\title{
PERCEPCIÓN SOBRE USO DE ALCOHOL Y TABACO EN FAMILIARES DE NIÑOS ENFERMOS. CONSULTA EXTERNA DEL HOSPITAL MATERNO INFANTIL DEL GUASMO SUR. GUAYAQUIL - ECUADOR ${ }^{1}$
}

Loor EA, Galera SAF. Percepción sobre uso de alcohol y tabaco en familiares de niños enfermos. Consulta externa del hospital materno infantil del Guasmo Sur. Guayaquil - Ecuador. Rev Latino-am Enfermagem 2004 março-abril; 12(número especial):3404.

Las enfermeras siempre deben preguntar a sus clientes sobre problemas con uso abusivo de alcohol en sus familias, independientemente de la especialidad de la profesional. El objetivo de esta investigación fue describir la concepción de familiares de niños pequeños con problemas de salud atendidos en un servicio de consulta externa sobre el hábito del uso del alcohol y el tabaco. Se entrevistó a 9 familiares y las entrevistas fueran analizadas bajo el análisis del contenido. La existencia de alcohol en la familia afloró como una queja. De 9 entrevistadas 6 tienen en diferentes generaciones presencia de consumidores de alcohol y tabaco. Las entrevistadas que identificaron conductas recurrentes de abuso de consumo de alcohol y tabaco utilizaron recursos auto correctivos buscando la estabilidad de un cambio dinámico intrafamiliar, sin éxito. El problema principal es la falta de autoridad de las mujeres, típica de la familia latinoamericana.

DESCRIPTORES: familia; uso del alcohol y tabaco; estúdio cualitativo; enfermería

PERCEPTIONS OF ALCOHOL AND TABACCO USE AMONG FAMILY MEMBERS OF ILL CHILDEN. EXTERNAL SURVEY BY THE MATERNALINFANT HOSPITAL OF GUASMO SUR. GUAYAQUIL - EQUADOR

Nurses must always ask its clients about problems with the abusive use of alcool in its families, independently of the professional speciality. The Objective of this study was to describe the conception of the familly members of young children with problems of health that attend to an ambulatorial service about the habit of alcool and tobacco use. Nine familly members were interviewed and the interviews had been analyzed through the content analysis. The existence of alcool in the family arose as a complaint. Six of the nine families have consumers of alcool and tobacco in different generations. Interviewees that had identified recurrent behavior of alcool and tobacco consumption abuse had searched resources to stabilize and the change intrafamiliar dynamic without sucess. The main problem is the lack of authority of the women, typical of the latinoamerican family.

DESCRIPTORS: family; use of alcohol and tabaco; qualitative research; nursing

PERCEPÇÃO SOBRE O USO DE ÁLCOOL E TABACO ENTRE OS FAMILIARES DE CRIANCAS DOENTES. CONSULTA EXTERNA DO HOSPITAL MATERNO-INFANŢIL DE GUASMO SUR. GUAYAQUIL-EQUADOR

Enfermeiros devem sempre perguntar a seus clientes sobre problemas com o uso abusivo de álcool em suas famílias, independentemente da especialidade profissional. O Objetivo desta investigação foi descrever a concepção de familiares de crianças pequenas com problemas de saúde atendidos em um serviço ambulatorial sobre o hábito do uso de alcool e tabaco. Foram entrevistados 9 familiares e as entrevistas foram analisadas pela análise de conteúdo. A existência de alcool na família aflorou como uma queixa. De 9 entrevistadas, 6 tem consumidores de alcool e tabaco em diferentes gerações. As entrevistadas que identificaram condutas recorrentes de abuso de consumo de alcool e tabaco utilizaram recursos buscando a estabilidade de uma mudança dinâmica intrafamiliar sem exito. O principal problema é a falta de autoridade das mulheres, típica da família latinoamericana.

DESCRITORES: família; uso de álcool e tabaco; pesquisa qualitativa, enfermagem

\footnotetext{
${ }^{1}$ Las opiniones expresadas en este articulo son de responsabilidad exclusiva de los autores y no representan la posición de la organización donde trabajan o de su administración; ${ }^{2}$ Docente de la Escuela de Enfermería de la Universidad de Guayaquil; ${ }^{3}$ Docente de la Escuela de Enfermería de Ribeirão Preto, de la Universidad de São Paulo, Centro Colaborador de la OMS para el desarrollo de la investigación en enfermería, correo electrónico: sugalera@eerp.usp.br
} 


\section{INTRODUCCIÓN}

El alcohol y el tabaco son drogas lícitas cuyo consumo es mundial. A su consumo cada día entran grupos de niños menores de edad, lo cual está comprometiendo el futuro de la sociedad. El daño orgánico que provocan en el individuo es evidenciado a través de múltiples estudios, así como también el daño a la familia, a la sociedad y al ecosistema ${ }^{(1)}$.

Los parientes en primer grado de alcohólicos tienen 7 por más probabilidades de desarrollar problemas asociados al abuso de alcohol que la población general ${ }^{(2)}$. Por eso las enfermeras siempre deben preguntar a sus clientes sobre problemas con uso abusivo de alcohol en sus familias, independientemente de la especialidad de actuación.

Es importante evaluar el efecto que el alcohol pudo haber tenido en generaciones anteriores de la familia y reconoce su relevancia para las cuestiones actuales de diferenciación individuales y familiares ${ }^{(3)}$. El sistema familiar y sus interacciones son mirados como un sistema social abierto, dinámico, dirigido a metas y autorregulado, por lo tanto no se lo describe en términos estáticos y estructurales, puesto que sus partes se encuentran dinámicamente relacionadas entre si con el entorno. Las cualidades de la familia surgen de la interacción de sus componentes y por lo tanto son emergentes ${ }^{(4)}$.

La finalidad de este trabajo es focalizar la dinámica interaccional del sistema familiar con relación al problema de uso de alcohol y de tabaco, desde el punto de vista de la familia. Este trabajo intenta aproximar más nuestro lente, tratando de incluir el punto de vista de otras personas además de la percepción del profesional y considerarlo a este último también dentro del sistema terapéutico. Por eso pensamos en entrevistar personas de la comunidad que estuviesen próximas de la primera investigadora, en una institución publica de consulta externa en el área de pediatría. Adoptamos el referencial sistémico para evaluación de situación de riesgo en las familias que están pasando por la fase de vida de cuidar a un niño pequeño.

No existe ningún estado que provoque cambios más profundos o que signifique desafío mayor para la familia nuclear o ampliada que la suma de un nuevo niño al sistema familiar ${ }^{(5)}$. El nacimiento presupone la ampliación de las responsabilidades paternas y maternas, el aumento de gastos familiares y el aparecimiento de innumerables situaciones de estrés, tales como las enfermedades y los accidentes infantiles. La familia frente a situaciones estresantes que superen los própios recursos se pueden constituir en alvos vulnerables al consumo abusivo de alcohol y tabaco. El estado del desenvolvimiento de la familia y el estado del desenvolvimiento del individuo, se interactúan para tornarse un contexto en que un problema como el alcohol, talvez sea tanto la causa como el efecto de la disfunción ${ }^{(1)}$.

\section{OBJETIVO}

El objetivo de esta investigación fue describir la concepción de familiares de niños pequeños con problemas de salud atendidos en un servicio de consulta externa sobre el hábito del uso del alcohol y el tabaco.

\section{MÉTODO}

Para desarrollar el presente estudio se escogió el abordaje cualitativo para guiar los procedimientos de recolección y análisis de datos. Fue utilizado el texto Análisis de Contenido de Bardin ${ }^{(6)}$.

Participantes

Hicieron parte del estudio nueve familiares de niños de un mes a 4 años, que acudieron a la consulta ambulatoria en el Hospital Materno Infantil del Guasmo Sur. El número de entrevistadas fue constituido por una muestra de conveniencia determinada por las condiciones del trabajo de investigación. La característica de la muestra, que no podía variar, era el que pertenecieran a esa área de influencia y que tuvieran ficha médica en dicha unidad y que su familia fuera de tipo extensa.

Local

El Hospital Materno Infantil del Guasmo Sur fue utilizado para entrar en contacto con los familiares. La sala de espera postpreparación de enfermería y preconsulta médica, en un área reservada contigua a los consultorios médicos y al área de preparación de enfermería, fue utilizada para la realización de las entrevistas.

Recolecta de datos

Se utilizó la entrevista semi-estructurada con 
referencia sistémica. En la entrevista semiestructurada, se aplicó un rotero de entrevista con referencial sistémico, el mismo contemplaba también la construcción del genograma. Las entrevistas ocurrieron en local aislado y fueron grabadas en cinta $K 7$, con duración de en promedio de 20 minutos. El período de recolección de informaciones se extendió del 31 de Julio al 30 de Agosto del 2002.

Análisis de los datos

La trascripción de las entrevistas, incluyendo la intervención de la investigadora más los datos y la confección del genograma, tomados en el momento de la entrevistas, constituyeron el documento madre sobre el cual se centró todo el trabajo.

Las entrevistas fueron transcritas y leídas cuidadosamente. En seguida fue escrito un enunciado sobre cada respuesta. El enunciado fue numerado de acuerdo con un código creado para identificar el sujeto y preservar su identidad. Los enunciados fueron leídos nuevamente y las palabras claves fueron subrayadas. A continuación se procedió al agrupamiento de los enunciados según sus semejanzas. Este agrupamiento fue impreso y se hizo una nueva lectura. El objetivo fue intentar construir un nuevo agrupamiento. La categorización temática se confrontó con el respaldo teórico, lo que dio confiabilidad a los resultados.

Rigor

Inicialmente presentamos la propuesta de validar el análisis de datos con los entrevistados proponiendo un segundo encuentro. Pero, debido a la baja concentración de consultas por año de 1.3/6 se optó por adjuntar al trabajo un resumen de todas las entrevistas. Los cuidados durante la entrevista para recolección de dados se direccionaron a procurar establecer un relacionamiento cordial, sin critica y confrontación. Los procedimientos de análisis fueron realizados en conjunto, investigador e supervisor, a través do correo.

\section{Aspectos éticos}

Inicialmente la primera investigador negoció con la Institución, solicitando permiso para utilizar su espacio físico para encontrar los participante más adecuados para la investigación. A continuación se providenciaron los tramites para obtener la aprobación del proyecto por la
Subdecana de la Facultad de Ciencias Médicas de la Universidad de Guayaquil. Todas las entrevistadas fueron informadas sobre el objetivo de la investigación, la importancia de su colaboración pero también de sus derechos de negarse a participar o de retirarse del estudio en cualquier momento. Además, fue esclarecido que la investigación no causaría daños y podría ser un momento para que las personas pudieran conversar sobre sus problemas. Todas las participantes del estudio firmaron el término de consentimiento informado y aceptaron colaborar con el proyecto.

\section{RESULTADOS Y DISCUSIÓN}

\section{Características generales de las entrevistadas}

Los 9 familiares entrevistados son residentes del Guasmo Sur, barrio urbano marginal de la ciudad de Guayaquil. la edad de los familiares varió entre 20 y 56 años. Los problemas de salud por lo que llevaron a la consulta al niño fue por problemas respiratorios, fiebre y mal estar general.

Las famílias pertenecieron al tipo "família ampliada"; esta está compuesta por tres generaciones, el estado civil que predominó fue la unión libre y de condición separados, sólo hubo una casada y separada. Solamente en un caso hubo la participación del padre del niño, en los otros la madre o la abuela acompañaban al niño.

Las familias que declararon no tener miembros consumidores de alcohol y tabaco identificaron más los problemas de salud asociados al uso de éstos, mientras que en las familias que informaron tener miembros consumidores el rechazo fue más enfático. Los daños los asociaron con violencia, daños a terceros, entre ellos principalmente la familia y los hijos y también consideraron el uso de las sustancias, una adicción.

Sin embargo, la demostración del rechazo al alcohol y el tabaco, también, por parte de las familias que no declararon tener miembros con consumo asiduo de alcohol, nos dejan entrever que no siempre el aprendizaje sobre los efectos de éste es por vivencias dentro de casa, pues estavan cientes de la existencia y lo identificaron como dañino.

Salud del niño y su contexto familiar

Ante la enfermedad del niño los familiares 
respondieron, generalmente, con preocupación e intervención compartida por parte de las tías y tío mayores de edad y mujeres básicamente. Incluso parecia ampliarse a los abuelos del menor que también manifiestaron su preocupación y solidaridad.

En la relación de pareja la estabilidad del hogar se muestraba afectada; la mayoría eran mujeres separadas y havia quejas de conflictos por consumo de alcohol y tabaco en las parejas que aún estavan juntos, a nivel de miembros mayores del núcleo familiar reflejavan más bien demostración de solidaridad y acuerdos en repartición de tareas frente a la crisis del niño enfermo.

El eje de la preocupación y el estrés que nace en cada uno de ellos, es en el momento que les toca visitar al médico y tener que enfrentar la desventajosa realidad económica y ver si pueden curar al infante y solventar los gastos de las medicinas, ya que esto corre por su cuenta, la consulta médica es gratuita. La población estudiada respondió con solidaridad y unión, "compartir, aunar esfuerzos económicos" básicamente, como dicen ellos.

También les embarga la curiosidad de saber de qué se trata la enfermedad del niño, saber su gravedad y el riesgo de complicarse, ya que estas situaciones las relaciona a más gastos económicos para los cuáles no se siente preparada, por que como dicen ellos:

... hay veces cuando no hay para la comida..., cuando "pás" se me enferma el bebe, se me enferman los dos bebes, ... que ya un lápiz que los dos dólares del mes para la escuela y no tenemos..., ...me dan la mano...

Interactuando con el alcohol

La existencia de alcohol y tabaco en la familia fluyó espontáneamente de las entrevistadas, no fue requisito de inclusión, más bien afloró como una queja de su presencia en el núcleo familiar o en su familia de origen.

De los 9 familiares entrevistados, 6 tenían en diferentes generaciones presencia de alcohol y tabaco en la familia. El patrón de respuestas sobre diversos temas de la entrevista se muestro muy similar al referirse a los diferentes comportamientos de consumo en las familias.

En todas ellas se manifestó la preocupación y solidaridad ante la enfermedad del infante, repudio por parte de las entrevistadas respecto al alcohol y el tabaco, observaciones de problemas en el individuo, familia y la sociedad por el abuso del consumo del alcohol más que por el tabaco.

La percepción del consumo de alcohol como factor genético y, como patrón de conducta en su núcleo familiar fue una explicación que la familia dió al origen del consumo del alcohol.

Describieron los patrones de consumo de alcohol observados a través del tiempo, en sus familias de origen o en las familias de su esposo, la experiencia la han vivido o la viven como una realidad negativa.

No rechazaron en ninguno de los casos al consumidor del alcohol y tabaco, sí se reniegaban frente a ellos, sí rechazaban al alcohol y al tabaco. Sobre el tabaco hasta les "hiede" y les parecia fuerte, les provocava reacciones de destrucción, quisieran que él no existiese, y sobre el alcohol deseaban que en las propagandas de la TV resaltasen el daño que hace y no la nueva marca que sacan a la venta.

El alcoholismo y el consumo de tabaco para las familias parece ser un problema, perciben que el daño que éstos provocan es a nivel individual, familiar y social.

El alcohol a nivel individual, hace daño en el organismo, su hígado, riñones, cerebro, mente; a nivel familiar causa problemas de interacción básicamente con su esposa, mal trato y también con sus hijos; y a nivel social perciben que provoca accidentes de transito, los hace pelear, golpearse, los botan del trabajo, tienen accidentes en sus trabajos.

El tabaco, lo identifican con el daño a nivel de pulmones del fumador y con el humo hace daño a los niños con asma, pero lo que más pareció causar mayor preocupación fue si alguno fuma alguna otra cosa que no solamente cigarrillo puede cometer cualquier barbaridad.

Las entrevistadas relataron que el alcohólico justifica su consumo, argumentando "que toma por que tiene problemas con la pareja”, pero ellas más bien creen que "el problema lo tiene por tomar". También observaron que el problema está "en la persona", que "él debería resolver sus problemas hacia adentro", que "él está mal". Y que "cuando consuma lo haga fuera de la visibilidad de los niños".

También consideraron que la trasmisión de creencias de generaciones anteriores influencian el consumo en su familia actual. Otras familias declararon que el consumidor lo hace inicialmente "por que le gusta" y "busca liberación y desfogarse de sus problemas" y luego sigue tomando, "se hacen adictos". 


\section{CONCLUSIONES}

Con las revelaciones espontáneas que las entrevistadas hicieron sobre el consumo de alcohol y el tabaco en la familia, reflejan que el problema lo tienen y lo sienten a flor de piel, así como también se observa que recargan el problema básicamente en el hombre. Las mujeres de Latinoamérica reciben grande prestigio por el rol de madre.

Frente a la problemática del consumo del alcohol y el tabaco, las familias han utilizado y siguen utilizando sus recursos, de ellos el que ha primado es la palabra, a través del consejo, recursos que no les ha surtido efecto. Reconocen que el problema no es sólo del consumidor. Entienden que es todo un sistema de consumo, ven como factores intervinientes los amigos e identifican que la familia de origen también tiene su aporte en esta conducta de consumo.

Entre las participantes de este estudio parece que la difícil situación económica frente a un niño enfermo, estimula sentimientos de solidaridad y unión entre los miembros mujeres y mayores del sistema familiar. También no se identifican disfuncionalidad en la pareja por la enfermedad del niño y ni tampoco por estar atravesando a la vez en la familia los cambios de tener un niño pequeño, pero sí dicen directamente que los problemas de las parejas que aún están juntas (3 de 9) es por el consumo del alcohol y tabaco de parte del marido.

Los resultados inesperados de alcoholismo y de violencia referidos por la familia, sin estar en los objetivos del trabajo, obliga a recomendar que la enfermera en sus parámetros de evaluación cotidianas considere la existencia de alcoholismo, tabaquismo y violencia en la familia y busque identificar su presencia a través de instrumentos adecuados.

Estes resultados sugieren que el papel de la enfermera en el contexto estudiado deve incluir la detección precoz de problemas relacionados al uso problemático de alcohol y tabaco. Las entrevistas realizadas en este estudio nos ofrecieron informaciones importantes, por eso acreditamos que la introducción de investigaciones sistematizadas con base en rotero estructurado podría ser útil y aplicable. Por lo tanto se propone dar continuidad al estudio para comenzar a evaluar la utilidad y aplicabilidad del rotero utilizado.

\section{AGRADECIMIENTOS}

Agradecemos a todos los docentes de la Escuela de Enfermería de Ribeirão Preto y a los treinta y tres participantes de nueve países latinoamericanos que participaron en el proceso de implementación de los programas, y también al Gobierno del Japón y al Programa de Becas de la OEA por su apoyo financiero y becas que posibilitaron la implementación del "I Programa Regional de Capacitación en Investigación para Enfermeros de América Latina".

\section{REFERENCIAS BIBLIOGRÁFICAS}

1. Krestan J, Bepko C. A família e o problema do alcoolismo. In: Carter BY, Mcgoldrick M, organizadoras. As mudanças no ciclo de vida familiar. Porto Alegre (RS): Artes Médicas; 2001. 2. Jerônimo DVZ, Pimenta AMC. Filhos de pais alcoolistas. Prorgama e Resumos da Jornada de Saúde Mental; 2000. Ribeirão Preto; 2000.

3. Wright L, Leahey M. Enfermeiras e Familias: um guia para avaliação e intervenção com famílias. São Paulo (SP): Editorial Roca; 1987.

4. Carlfred B, Garcia E, Gonzalo M. Psicología social de la Familia. Barcelona: Ed. Paidos; 2000.

5. Bradt JO. Tornándose Padres: Familias con hijos pequeños. In: Carter BY, Mcgoldrick M, organizadoras. As mudanças no ciclo de vida familiar. Porto Alegre (RS): Artes Médicas; 2001. 6. Bardin L. Análisis de Contenido. Francia: Ediciones 70; 1977. 